\title{
De la metropolización a las agrópolis. El nuevo poblamiento urbano en el Chile actual
}

\author{
Alejandro I. Canales \\ Universidad de Guadalajara, Guadalajara, México. Email: acanales60@gmail.com

\section{Manuel Canales Cerón} \\ Universidad de Chile, Santiago, Chile. Email: mcanalesc@gmail.com
}

\begin{abstract}
Resumen: La modernización del agro chileno ha generado nuevos patrones de asentamiento y movilidad de la población tanto en ámbitos locales y regionales, como a nivel nacional. El tradicional modelo de desarrollo urbano-metropolitano, ha sido sustituido por un modelo de desarrollo agropolitano, el cual ya no se sustenta en el crecimiento y metropolización del país, sino en el crecimiento de un amplio abanico de ciudades agrarias. En este artículo se analiza la dinámica demográfica de estas ciudades agrarias. Proponemos el concepto de agrópolis, el cual lo definimos como aquellos espacios en los que la actividad económica predominante está vinculada directamente al agro, ya sea como actividad primaria (agricultura), como actividad secundaria (agroindustria) o terciaria (comercio y servicios orientados a la agricultura y/o la agroindustria). Este concepto de agrópolis nos permite superar los vacíos de la dicotomía rural-urbana, proponiendo una oposición conceptual más amplia y compleja, entre lo que serían las agrópolis y las metrópolis.
\end{abstract}

Palabras clave: agrópolis, ciudades medias, modernización agraria, urbanización, población y territorio.

\section{From metropolization to agropolis. The new urban settlement in Chile today}

\begin{abstract}
The agrarian change in Chile has created new settlement patterns andpopulation mobility in local and regional level, as well as in national level. The classic model of metropolitan urbanization has been replaced by an agropolitan development model, which is supported by the growth of a wide range of agricultural towns. This article analyzes the population dynamics of these agricultural cities. We propose the concept of agropolis, which we define as those spaces in which the predominant economic activity is directly linked to agriculture, either as a primary activity (agriculture), as a secondary activity (agribusiness) or tertiary (trade and service-oriented agriculture and/or agrobusiness). This concept allows us to overcome the gaps in rural-urban dichotomy, proposing a conceptual opposition broader and more complex, between what would be the agropolis and the metropolis.
\end{abstract}

Key words: agropolis, intermediate-size city, agricultural modernization, urbanization, population and territory. 


\section{Desde metropolização as Agropolis. $O$ novo assentamento urbano no Chile}

Resumo: A modernização agrícola chileno criou novos padrões de assentamento e mobilidade da população em nível local e regional, como a nível nacional. O modelo tradicional de desenvolvimento urbano- metropolitano, foi substituído por um modelo de desenvolvimento agropolitano, o que já não é suportado pelo crescimento e metropolização do país, mas no crescimento de uma vasta gama de cidades agrícolas. Este artigo analisa a dinâmica da população dessas cidades agrícolas. Propomos o conceito de Agropolis, que definimos como aqueles espaços em que a atividade econômica é predominante e diretamente ligada à agricultura, seja como atividade primária (agricultura), como uma atividade secundária (agronegócio) e terciária (comércio e serviços orientados para agricultura ou agronegócio). Este conceito de Agropolis nos permite superar as lacunas na dicotomia rural-urbana, propondo uma oposição conceitual mais ampla e complexa, entre o que seria o Agropolis e metrópoles.

Palavras-chave: Agropolis, cidades médias, modernização agrícola, urbanização, população e território.

\section{Introducción}

El modelo económico chileno, basado en la modernización y globalización del agro ha generado profundos cambios no sólo en la organización económica y social de la agricultura y de sus modos productivos, sino también en las estructuras territoriales de la dinámica demográfica, especialmente en lo que se refiere a los nuevos patrones de asentamiento y movilidad de la población tanto en ámbitos locales y regionales, como a nivel nacional (de Mattos, 1998). El tradicional modelo de desarrollo urbano-metropolitano, ha sido sustituido por un modelo de desarrollo agropolitano, el cual a diferencia del primero, ya no se sustenta en el crecimiento y metropolización del país, sino en el crecimiento de un amplio abanico de ciudades agrarias, las que se convierten en los centros urbanos sobre los que gravita el actual desarrollo agrario chileno (Canales, M., 2008).

Este proceso de expansión urbana de ciudades agrarias, muestra también una importante diferencia con el modelo clásico de expansión de ciudades medias con base en procesos de relocalización industrialcomo los que se han dado en otros países latinoamericanos (Sobrino, 2002; Aguilar, Graizbord y Sánchez, 1996). A diferencia de estos, en el caso chileno el centro económico y productivo de la reconversión económica, no está en la ciudad en sí misma, sino en territorio agrario. La ciudad media eneste sentido, es la forma urbana que se dinamiza a partir de la reconversión productiva del agro, y de su inserción en la economía global. Por eso mismo, se trata de la expansión de agrourbes, y no de ciudades medias en general (Canalesy Hernández, 2011; PNUD, 2008). 
Considerando lo anterior, en este texto se analiza la dinámica demográfica de estas ciudades agrarias. Al respecto, proponemos el concepto de agrópolis, el cual lo definimos como todos aquellos espacios en los que la actividad económica predominante está vinculada directamente al agro, ya sea como actividad primaria (agricultura), como actividad secundaria (agroindustria) o terciaria (comercio y servicios orientados a la agricultura y/o la agroindustria). Asimismo, este concepto de agrópolis, o territorios agrarios, nos permite ir más allá de los vacíos que actualmente esta dejando la dicotomía rural-urbana, proponiendo en cambio, una oposición conceptual más amplia y compleja, entre lo que serían las agrópolis y las metrópolis.

\section{Urbanización y metropolización en Chile y América Latina}

América Latina es junto a América del Norte, la región más urbanizada del planeta. Hacia el 2010 se estimaba que prácticamente el $80 \%$ de su población residía en localidades urbanas, cifra ligeramente inferior a la de USA y Canadá, pero muy superior a la de África y Asia, donde aún más del $50 \%$ de sus poblaciones reside en localidades rurales. Asimismo, cabe señalar que la urbanización es un fenómeno relativamente reciente en la región, y se desarrolla principalmente a partir de los años cincuenta del siglo pasado. En efecto, hasta 1950, América Latina era aún un continente predominantemente rural (Rodríguez, et al, 2011).

Diversos autores identifican al menos dos grandes etapas en el proceso de urbanización en la región. Una primera, hasta mediados de los setenta, con base en el modelo de crecimiento hacia adentro, y una segunda, en el marco de las transformaciones urbano-regionales derivadas de la globalización económica (Cunha y Rodríguez, 2009; De Mattos, 2002).

La primera etapa, que abarca de los años cincuenta hasta mediados de los setenta, se caracteriza por la concentración de la población en grandes ciudades y zonas metropolitanas, constituidas en su gran mayoría por la ciudad capital de cada país, extendiéndose en algunos casos a la segunda y tercera ciudad más importante. Es lo que podríamos denominar como crecimiento urbano-metropolitano, el cual se sustentó en el impulso de un modelo de desarrollo e industrialización sustitutivo de importaciones, con una participación activa y predominante del Estado (Garza, 2003).

La concentración metropolitana favorecía la generación de economías de escala y de aglomeración necesarias para el impulso de la industrialización, pero a la vez, generaba también no pocas desigualdades y desequilibrios regionales, tanto del tipo campo-ciudad, como entre las zonas metropolitanas y las otras ciudades, las que por su tamaño y dinamismo, no disponían de las mismas condiciones para la atracción de inversiones, industrias y población (Pradilla Cobos, 1993). 
Asimismo, este modelo de urbanizaciónmetropolitana se ve favorecido por el contexto demográfico. Estas décadas se corresponden con las primeras etapas de la Transición Demográfica en la región, caracterizadas por el incremento en los niveles de crecimiento natural de la población (Villa, 1992). Esto favorece la generación de excedentes demográficos, especialmente en zonas rurales, (donde tradicionalmente se da una mayor natalidad y fecundidad) y su movilidad desde el campo hacia las metrópolis y en menor medida, otras ciudades y áreas urbanas (Rodríguez y Busso, 2009).

Hacia mediados de los setenta, este modelo de industrialización y urbanización metropolitana, muestra signos de agotamiento, derivado tanto de las limitaciones propias del modelo sustitutivo deimportaciones, como de los cambios que simultáneamente se estaban generando en la economía mundial (CEPAL, 2012). En este sentido, la restructuración productiva, en el marco de la globalización de la economía latinoamericana, tuvo importantesimpactos tanto en la localización de las actividades económicas y productivas más dinámicas, como en los patrones de migración y distribución territorial de la población (Atienza y Aroca, 2012; Diniz y Crocco, 1998).

Aunque la situación muestra grandes diferencias entre los diferentes países de la región, en algunos casos la expansión urbana se desplaza desde las principales zonas metropolitanas hacia el auge y crecimiento de las llamadas ciudades medias. En esta dinámica, se conjugan diversos procesos muy distintos, entre los cuales destacan por un lado, la reducción y descentralización del aparato estatal (concentrado en gran medida en la ciudad capital), y por otro, los nuevos patrones de localización industrial, en el marco de la globalización de los procesos productivos (Cunha, 2002; Lufin Varas y AtienzaUbeda, 2010).

La globalización de la economía mundial, promueve nuevos patrones de localización industrial que no siempre favorecen a las ciudades metropolitanas, promoviendo en cambio, un proceso de expansión urbana que incorpora diversas ciudades medias a los nuevos modelos de industrialización orientada ahora ala exportación y participación de los procesos productivos globales y de la nueva división internacional del trabajo (Hiernaux, 1998). Es el caso de México, por ejemplo, en donde a través de la industria maquiladora de exportación, las ciudades de la frontera norte así como las del centro y occidente del país, reciben el impulso de la inversión extranjera que promueve la localización de industrias y segmentos del proceso de producción global (Alegría, Carrillo y Alonso, 1997).

Estos procesos se acompañan de cambios en los patrones de movilidad y migración interna en cada país. Por un lado, se reduce la intensidad y magnitud de estos flujos (Rodríguez, et al, 2011), a la vez que por otro lado, emergen nuevas pautas de migración. En este sentido, el tradicional flujo campo-metrópolis, es sustituido por la emergencia de nuevas pautas de movilidad, entre las que se destacan los desplazamientos entre ciudades 
(migración urbana-urbana), la emigración neta de las ciudades capitales y zonas metropolitanas, así como diversas formas de movilidad rural-rural (Rodríguez y Busso, 2009).

El caso de Chile, sin embargo, presenta ciertas particularidades que nos interesa analizar. Por un lado, si bien hasta los años setenta Chile reproduce a grandes rasgos el patrón de urbanización y metropolización ya descrito, éste sin embargo se inicia en forma mucho más temprana que en la mayoría de los demás países de la región, de tal modo que ya hacia la primera década del siglo XX, más del 40\% de la población residía en localidades urbanas. Asimismo, la concentración de población en la ciudad principal (Santiago), a la vez que se inicia antes que en otras capitales de América Latina, es además significativamente mayor al promedio regional. En el 2002, en la ciudad de Santiago residían más de 5.4 millones de personas, lo que representaba más del 36\% de la población del país ${ }^{1}$.

Por otro lado, y este es el aspecto que se nos hace más relevante y que constituye el centro de nuestra atención, la dinámica de expansión de ciudades medias, si bien reproduce la tendencia general a una relativa desconcentración metropolitana, presenta sin embargo, significativas diferencias en cuanto a las formas urbanas que adopta, y en particular, a las bases económicas y demográficas que la sustentan (Rodríguez, et al; 2009).

En efecto, hasta mediados de los setentas, Chile como gran parte de los países de la región, seguía el patrón general de desarrollo con base en la sustitución de importaciones. Esta era la base estructural que explicaba en gran medida, el proceso de urbanización y concentración demográfica en la ciudad de Santiago, convirtiéndola en la gran metrópolis del país (Geisse, 1983).

Asimismo, desde mediados de los setentas, se inicia un proceso de expansión urbana sustentado en el crecimiento de ciudades medias, que entran a competir y en algunos casos, a sustituir el predominio de las grandes metrópolis. Sin embargo, a diferencia de lo que sucede en otros países como México, Brasil, Colombia, entre otros, en Chile esta segunda fase de expansión urbana está sustentada en una base económica y productiva diferente.

Mientras en algunos casos paradigmáticos, como México, la expansión de las ciudades medias se sustentó en la incorporación de esas ciudades a los circuitos globales de producción industrialconfigurando espacios de localización de determinados segmentos de la producción industrial globalizada, a través de la industria maquiladora, en particular (Soto, et al, 2008), en el caso de Chile, en cambio, la expansión urbana de las ciudades medias y pequeñas se da en el marco de un modelo económico y de transformaciónproductiva muy diferente.

En el caso de Chile, la transformación productiva del país, y su reinserción en la economía global, se sustentan básicamente, en la moder- 
nización y reconversión productiva de la estructura agraria y las actividades piscisilvoagropecuarias. Esta transformación, inicia a mediados de los setentas, anticipándose a los demás países de la región, y se sustenta precisamente en la reorientación de la base económica del país, transitando de la clásica estructura de la industria sustitutiva, al fomento y consolidación de una economía de agroexportación (Canales, 1996). Para ello, primero durante la dictadura militar, y posteriormente, con los gobiernos de la Concertación, se impulsó el desmantelamiento de la industria nacional, reorientando la inversión doméstica y atrayendo inversión extranjera hacia las actividades agrícolas, ganaderas, forestales y la industria pesquera (Gwinne y Kay, 1997).

En tal sentido, desde mediados de los setenta se impulsa un nuevo modelo económico, también sustentado en su vocación exportadora y de reinserción en la economía global, pero que a diferencia de modelos similares en otros países, en el caso de Chile se centra fundamental y casi exclusivamente, en la promoción de su base agroexportadora (Kay, 2002)².

Con base en este nuevo modelo económico, se reconfiguran y revierten en cierta medida, las tradicionales ventajas de localización y de atracción que caracterizan a las grandes ciudades, en este caso Santiago, Valparaíso y Concepción. Estas zonas metropolitanas no sólo pierden terreno en su capacidad de atracción de inversiones y actividades productivas, sino también y fundamentalmente de atracción de población y de sus flujos migratorios (Atienza y Aroca, 2012; Canales y Canales, 2012).

Ahora bien, después de más de 30 años de funcionamiento con relativo éxito de este modelo económico y productivo, podemos observar cómo él ha generado profundos cambios no sólo en la organización económica y social de la agricultura y de sus modos productivos, sino también transformaciones demográficas y territoriales no menos importantes, especialmente en lo que se refiere a los nuevos patrones de asentamiento y movilidad de la población tanto en ámbitos locales y regionales, como a nivel nacional (Berdegué, et al, 2011a; Canales y Hernández, 2011).

Estas tendencias ilustran un nuevo patrón migratorio, que rompe no sólo con el ya tradicional flujo campo-ciudad, sino también con el de la llamada transición urbana, que se manifiesta en flujos urbano-urbano, desde las metrópolis a las ciudades medias (Rodríguez y Busso, 2009). A diferencia de estos dos patrones de movilidad de la población, en este caso se trata de un proceso de relocalización de población al interior de espacios agrarios, que adopta la forma de una migración que proviene tanto del campo como de pequeños poblados y que se dirige hacia las ciudades agrarias, mismas que se desempeñan como las cabeceras urbanas de estos territorios agrarios. Se trata de un nuevo patrón de poblamiento sustentado en el auge de asentamientos agrourbanos, pero que no implican necesariamente, el rompimiento de los diversos vínculos sociales, económicos y demográficos con los demás poblados rurales y urbanos (PNUD, 2008; Canales, 1996). 
En efecto, desde los setentas, se habría iniciado en Chile proceso de repoblamiento agrario, el cual, a diferencia de los poblamientos agrarios del pasado, no estaría centrado en una territorialidad esencialmente rural, sino en la articulación de sistemas de poblados rurales y urbanos. Se trata de la configuración de una nueva territorialidad agraria, que en su espacialidad, articula pueblos rurales y ciudades agrarias de diversos tamaños y dinámicas (Berdegué et al, 2011b; Canales y Hernández, 2011).

Considerando lo anterior, nuestra tesis es que en el caso de Chile, la expansión urbana con base en el crecimiento de las ciudades medias, no estaría vinculado ni sería resultado de un proceso de descentralización y desconcentración metropolitana, o de relocalización industrial, en el marco de un proceso de transición urbana, sino que se debería al impulso de un modelo de urbanización completamente diferente, sustentado en la modernización productiva y globalización económica del agro chileno. En tal sentido, no es posible hablar, en el caso chileno, de ciudades medias en el sentido clásico de ciudades industriales, o similares, sino más bien, habría que referirse a estas como ciudades agrarias, esto es, como el componente urbano de los territorios agrarios en el Chile contemporáneo. Por lo mismo, no son ciudades que compitan directamente con las metrópolis en la localización industrial y de actividades de servicios y de apoyo, sino, que forman parte de una nueva configuración urbana, que se yuxtapone a la configuración urbano metropolitana tradicional ${ }^{3}$.

En este sentido, nuestra tesis es que en Chile se habría configurado un modelo de expansión urbana y de ciudades medias diferentes al esperado y que se observa en ciertos países de la región. Se trata del desarrollo de agrourbes, de un proceso de urbanización agraria, en el marco de la configuración de espacios agropolitanos. Al respecto, el siguiente esquema permite ilustrar esta tesis sobre la expansión agrourbana, como alternativo, al de la expansión urbana metropolitana, y/o a la expansión urbano-industrial.

\section{Ilustración 1.}

\section{Modelos de expansión urbana y de ciudades medias}

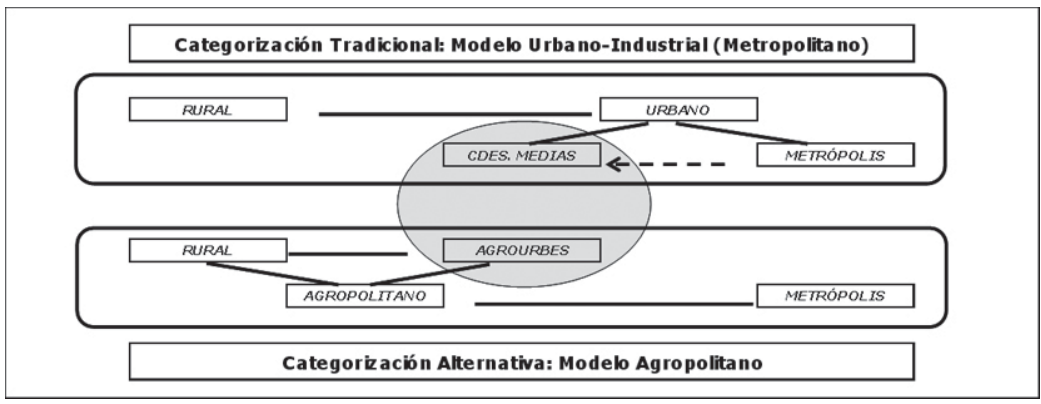

Fuente: Elaboración propia 
Con base en este esquema, proponemos diferenciar dentro de la tradicional categoría urbana, dos tipos estructurales y opuestos: una forma urbana metropolitana, y una forma urbana-agraria (agrourbes). En tal sentido, nuestra propuesta es que mientras el modelo urbano-metropolitano, se sustenta en la configuración de un sistema urbano que articula un poblamiento metropolitano con las ciudades medias, el modelo agropolitano en cambio, se sustenta en la articulación de un patrón de poblamiento rural (producción agro-primaria) con un patrón de poblamiento urbano (agrosecundario y terciario). Lo que define en ambos casos, es el predominio de la actividad agraria (Canales, M, 2008).

\section{Crecimiento demográfico y urbanización en Chile en el siglo XX}

Como en todos los países latinoamericanos, el siglo XX puede ser caracterizado como el siglo del crecimiento demográfico, mismo que resulta del avance en la transición demográfica en la región. En el caso chileno, la población pasó de 3.2 millones en 1907, a 15.3 millones en el 2000, y se estima que habría alcanzado los 17 millones en el 2010 ${ }^{4}$. Es decir, en estos últimos 100 años, la población prácticamente se habría quintuplicado. Sin embargo, esta dinámica presenta dos características que es necesario considerar.

Por un lado, no se trata de una tendencia lineal y uniforme a lo largo de todo el periodo, sino que podemos identificar al menos tres etapas, a saber:

- Una primera etapa, que abarcaría hasta los años 30s, cuando la población crece a tasas relativamente bajas, como consecuencia de los altos niveles de mortalidad imperantes en esos años.

- De los años 40s a los setentas, que corresponde a la etapa de mayor crecimiento demográfico, que alcanza su punto máximo en los 50s y 60s, con tasas del orden del 2.3\% anual. En esta fase, la población de Chile está en pleno proceso de Transición Demográfica.

- A partir de los setentas inicia el descenso del ritmo de crecimiento, alcanzando en la última década una tasa anual del 1\%. Corresponde a la última fase de la Transición Demográfica, la cual junto a una reducida fecundidad, está asociada también a un cambio en la estructura demográfica, y al envejecimiento de la población.

Una segunda característica de la dinámica demográfica en Chile, es que a pesar de haberse más que quintuplicado su población en los últimos 100 años, en el contexto regional se trata más bien de un crecimiento relativamente moderado. De hecho, desde mediados del siglo pasado, el ritmo de crecimiento demográfico de Chile, ha estado sistemáticamente por de- 
bajo del promedio latinoamericano, ubicando a Chile, entre los países de menor crecimiento relativo, junto a Argentina, Cuba y Uruguay, con los cuales comparte el hecho de presentar una Transición Demográfica temprana y de un ritmo moderado.

\section{Ilustración 2. \\ Chile, 1907-2010. Población y tasa anual de crecimiento intercensales.}

\begin{tabular}{|c|c|}
\hline Año & Población \\
\hline 1907 & $3,231,496$ \\
\hline 1920 & $3,728,563$ \\
\hline 1930 & $4,287,443$ \\
\hline 1940 & $5,023,539$ \\
\hline 1950 & $6,081,931$ \\
\hline 1960 & $7,643,277$ \\
\hline 1970 & $9,569,631$ \\
\hline 1980 & $11,174,173$ \\
\hline 1990 & $13,178,782$ \\
\hline 2000 & $15,397,784$ \\
\hline 2010 & $17,094,270$ \\
\hline $\begin{array}{l}\text { Fuentes: } 1907 \text { a 1940, INE, Censos } \\
\text { de Población. 1950 a 2010, INE, } \\
\text { Proyeccion es de población, } 1950- \\
\text { 2050. }\end{array}$ \\
\hline
\end{tabular}

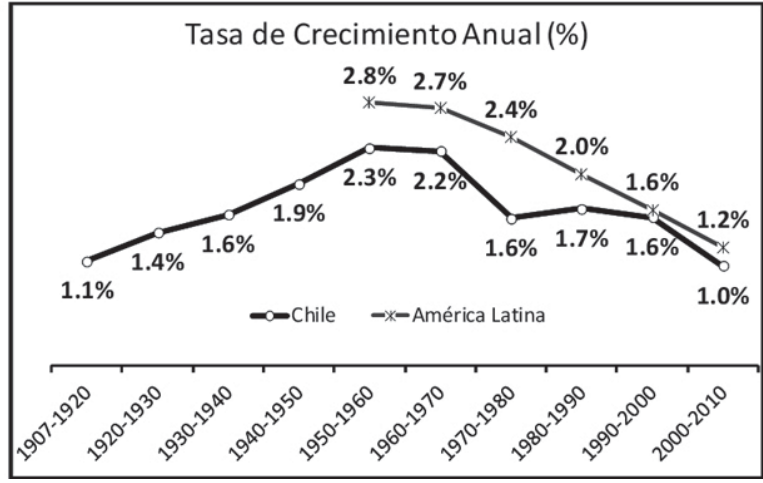

Fuentes: elaboración propia con base en Censos de Población, y proyecciones de población, INE, Chile. Y datos para América Latina tomados de CEPALSTAT. http://websie.eclac.cl/infest/ajax/cepalstat.asp?carpeta=estadisticas

El crecimiento demográfico estuvo acompañado de un proceso de urbanización y concentración espacial de la población. En Chile, la concentración territorial de su población, ha sido una constante histórica. Por factores de diversa índole (geografía, climas adversos, factores políticos y económicos, entre otros) desde siempre el asentamiento de la población chilena se ha concentrado en un tercio de su territorio, que corresponde básicamente a la Zona Central, que va desde Coquimbo por el Norte, hasta Chiloé y Puerto Montt por el Sur. Tanto la zona del Norte Grande y parte del Norte Chico, como de la zona Austral, han sido tradicionalmente territorios prácticamente desolados y aislados, en donde los asentamientos humanos se han impulsado como una estrategia geopolítica de poblamiento y ocupación del territorio.

Los datos así lo demuestran. Ya en 1907 más del 90\% de la población chilena se asentaba en la zona central, proporción que se mantiene más o menos estable a lo largo de todo el siglo XX. Sin embargo, dentro de este patrón de poblamiento, se inicia desde las primeras décadas del siglo pasado, un segundo proceso de concentración demográfica que adquiere dos formas complementarias. Por un lado, el crecimiento y concentración de la población en lo que es actualmente la Región Metropolitana de San- 
tiago ${ }^{5}$. Por otro lado, el proceso de urbanización, esto es, la concentración de la población en localidades urbanas.

En el primer caso, los datos son claros y elocuentes, e indican una tendencia a la concentración de la población en la RMS, la cual pasa de tener el 17\% de la población en 1907, al 40\% en el 2002, proporción que se estima se haya mantenido en el 2010. Podemos identificar al menos dos grandes etapas en este proceso de concentración regional.

- Por un lado, desde 1907 hasta 1970, aproximadamente, cuando se da una tendencia de crecimiento constante y persistente de la concentración, expresada en la proporción de la población que se asienta en la RMS.

- Por otro lado, desde los años setentas hasta la actualidad, cuando esta tendencia concentradora comienza a desacelerarse, para frenarse por completo en la primera década de este nuevo siglo.

\section{Ilustración 3. \\ Chile, 1907-2010. Distribución de la Población según grandes áreas geográficas, y Región Metropolitana de Santiago.}

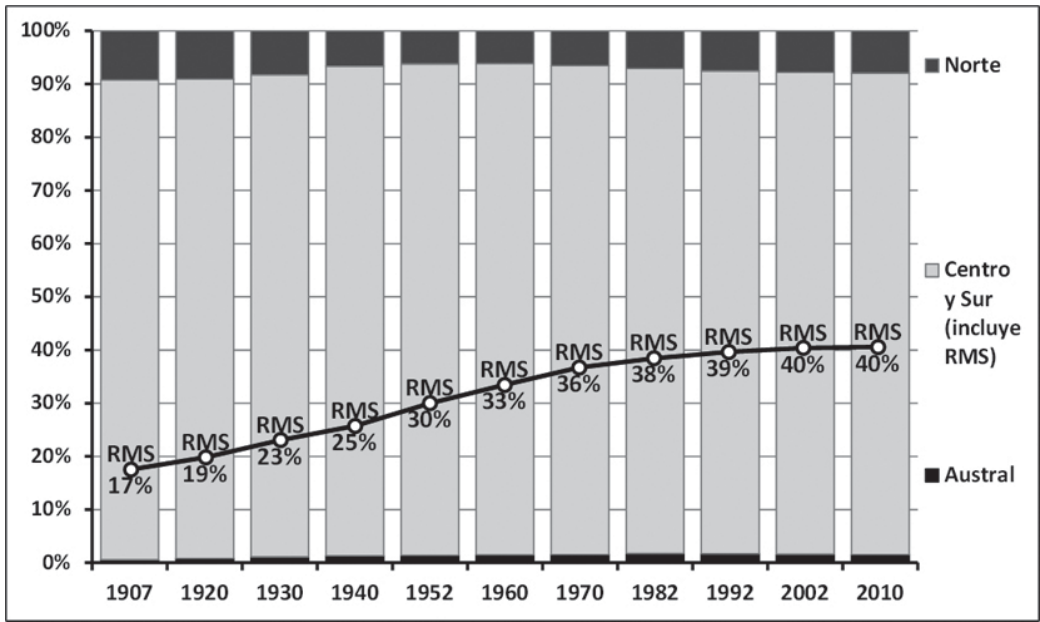

Fuentes: elaboración propia con base en Censos de Población, y proyecciones de población, INE, Chile.

Este proceso de concentración territorial de población en la RMS, es expresión de un fenómeno más complejo. Nos referimos a la modernización y urbanización de Chile, a partir del proceso de industrialización impulsado desde los años cuarenta. En efecto, como se ilustra en la gráfica, la 
población urbana en Chile pasó de 1.4 millones en 1907, a 13.5 millones en el 2010. Esto es, en 100 años, prácticamente se septuplicó.

Lo que resulta sin duda relevante, es que en el mismo período la población rural prácticamente se mantuvo estable fluctuando alrededor de los 2 millones de habitantes. De hecho, con excepción de 1907, nunca más la población rural ha descendido de este guarismo, aunque tampoco nunca ha superado la barrera de los 2.5 millones.

Ahora bien, sobre el proceso de urbanización, cabe señalar que desde siempre Chile ha presentado un grado de urbanización más temprana y superior al promedio de América Latina. Ya en 1907, prácticamente el 45\% de la población chilena residía en localidades urbanas, proporción que se incrementa al $87 \%$ en años recientes.

Asimismo, y al igual que la concentración regional, podemos identificar las mismas dos etapas en el proceso de urbanización, las cuales nos permitirán sustentar nuestra tesis de que cada una de ellas, corresponde en realidad a dos modelos de urbanización diferentes entre sí, no sólo en cuanto a sus formas, sino también en cuanto a sus fundamentos y bases estructurales que los generan.

i) Por un lado, una primera etapa que adopta la forma de concentración urbano-metropolitana, y que abarca desde los años 30s hasta inicio de los años setentas. Corresponde a la fase de industrialización basada en la sustitución de importaciones, misma que fomentó la concentración de la actividad manufacturera en las zonas metropolitanas (Santiago, Concepción y Valparaíso), así como de diversas actividades económicas vinculadas a la industria (transporte, comunicaciones, construcción, comercio y servicios). Este proceso de concentración económica, es la base de la concentración demográfica en las principales ciudades y metrópolis de Chile.

Esto se evidencia al comparar el ritmo de crecimiento de las tres ciudades metropolitanas (Santiago, Valparaíso y Concepción) con la del resto de localidades urbanas del país. Entre 1930 y 1970, estas tres ciudades crecieron en conjunto a una tasa anual promedio de 3.1\%, esto indica un ritmo de duplicación de cada 22 años, aproximadamente. Por el contrario, el resto de localidades urbanas, creció en promedio, a una tasa de $2.7 \%$.

Tomando en cuenta estos datos, y suponiendo un similar ritmo de crecimiento natural, estimamos que en este período, las ciudades metropolitanas concentraron el 63\% de la emigración neta rural, mientras que el resto de localidades urbanas, recibieron tan sólo el 37\% de este flujo migratorio.

ii) Por su parte, identificamos una segunda etapa en el proceso de urbanización, la cual se corresponde con el impulso y consolidación de un nuevo modelo económico, el cual no junto con promover el desmantelamiento de la industria sustitutiva de importaciones, y del rol del 
Estado en la economía, fomentó un proceso de modernización productiva del agro junto con el desarrollo y reconversión del sector agroindustrial, todo ello orientado a la inserción de Chile en la economía global, a través de la promoción de la agroexportación, tanto de productos primarios, como agroindustriales.

En esta fase, el proceso de urbanización no se sustenta ya en el crecimiento metropolitano, sino en el auge de ciudades medias y demás localidades urbanas. Efectivamente, entre 1970 y el 2002, el ritmo de crecimiento demográfico de las metrópolis prácticamente se desploma, cayendo a una tasa de sólo un 1.9\% anual promedio, muy similar al promedio nacional, que fue de $1.75 \%$. Por el contrario, el resto de las localidades urbanas, mantiene su alto ritmo de crecimiento, con una tasa del $2.5 \%$, muy por encima del promedio nacional.

Estas diferencias en el ritmo de crecimiento, se sustentan en una reorientación de los flujos migratorios provenientes de localidades rurales. Si en el pasado, las metrópolis eran las principales zonas de atracción, en esta etapa son las ciudades medias y pequeñas las principales localidades hacia donde se dirige la emigración rural. En efecto, estimamos que entre 1970 y el 2002, prácticamente 1.37 millones de personas habrían emigrado del campo a las ciudades medias y pequeñas y demás poblados urbanos no metropolitanos. Esto representa el 77\% de la emigración neta rural de este periodo. Por el contrario, las ciudades metropolitanas, recibieron tan sólo el 23\% de esta emigración neta rural, misma que corresponde aun volumen cercano a las 400 mil personas. En otras palabras, si en el pasado la emigración rural a las metrópolis era un $70 \%$ superior a la que iba a ciudades medias y pequeñas, actualmente, esta relación se invierte, de tal modo que la emigración rural a estas ciudades, es más de 3 veces superior a la que se dirige a las metrópolis.

Una forma sintética de ver esta diferente evolución de las metrópolis y demás localidades urbanas, es a través del Índice de Primacía Metropolitana, el cual mide la relación entre la población que residen en zonas metropolitanas, respecto a la que reside en las demás localidades urbanas del país. Como se observa en la siguiente gráfica, entre 1920 y 1970, este índice presenta un crecimiento sistemático, indicando con ello una creciente primacía de las ciudades metropolitanas, por sobre el resto de localidades urbanas. Corresponde a la etapa de la tradicional emigración campometrópolis, y en donde las ciudades medias y pequeñas conformaban un estrato relativamente estancado y atrasado.

Por el contrario, entre 1970 y el 2002, el índice de primacía metropolitano muestra el comportamiento inverso, reduciéndose sistemáticamente en cada década. Esto pone en evidencia el cambio en la dinámica de urbanización, la cual pasó de sustentarse en el desarrollo metropolitano, a sustentarse en el auge de ciudades medias y demás poblados urbanos, localidades que muestran un gran dinamismo económico y demográfico, y hacia las cuales se dirige actualmente el grueso de la emigración neta rural. 


\section{Ilustración 4.}

Chile, 1907-2002. Población urbana y rural, e indicadores demográficos de la urbanización

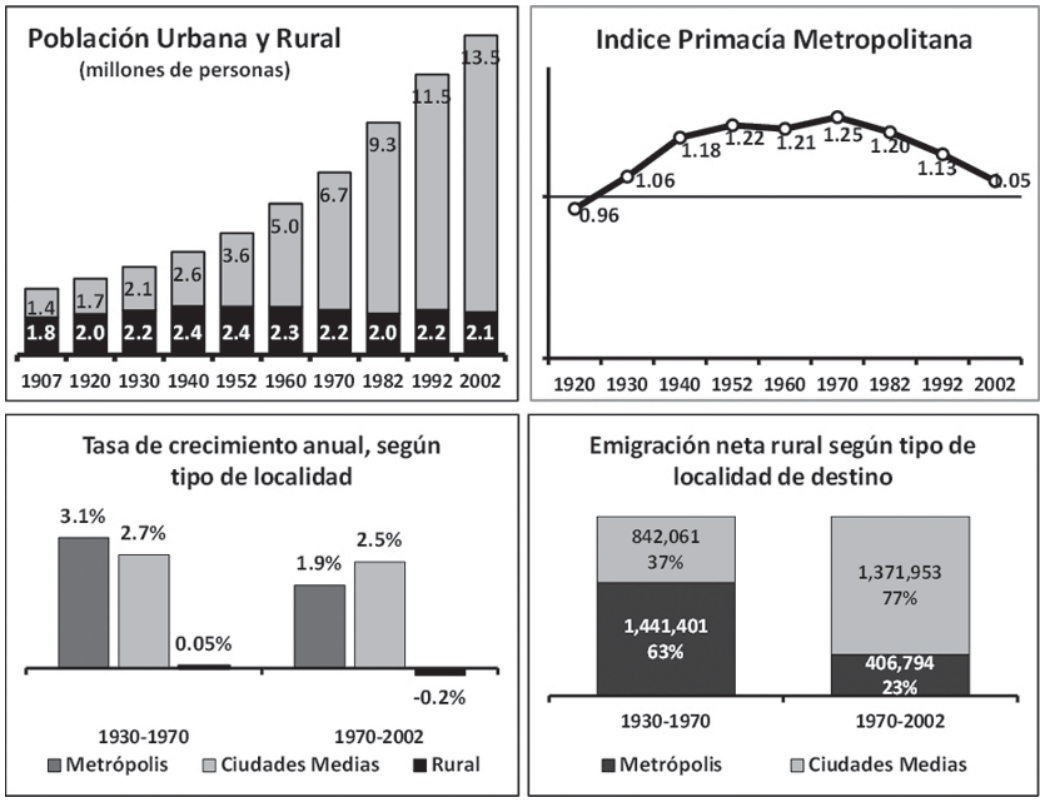

Fuentes: elaboración propia con base en Censos de Población, y proyecciones de población, INE, Chile.

\section{Reconversión agroindustrial y expansión urbana en Chile}

Este cambio en la forma que adopta la urbanización en Chile (con base en la expansión de ciudades medias y otros poblados urbanos, en contraposición al crecimiento metropolitano tradicional) es un fenómeno que también se ha manifestado en otros países de América Latina (Aguilar, Graizbord y Sánchez, 1996). Al respecto, suele documentarse el crecimiento de determinadas ciudades medias, a partir de sus ventajas competitivas frente a las nuevas pautas de localización industrial, derivadas de la nueva división internacional del trabajo, la cual, y que conlleva la segmentación y reubicación de las distintas fases del proceso industrial. En este marco, en el caso de México por ejemplo, vemos el auge de las ciudades de la frontera norte, así como de algunas urbes medianas del centro y occidente de país, a partir de la localización de diversas empresas en el marco del impulso a un proceso de industrialización basado en la maquila de exportación, esto es, en el ensamblaje de productos finales para su exportación (Alegría, Carrillo y Alonso, 1997). 
Sin embargo, en el caso de Chile, el crecimiento de las ciudades medias, parece tener otras raíces estructurales, que no se corresponden con factores de localización y atracción de determinados segmentos productivos de la actividad industrial y manufacturera global, esto es, de su hipotética inserción en lo que se ha denominado como una fábrica global. Por el contrario, en el caso chileno se trata más bien de una expansión urbana sustentada en la modernización productiva del agro y en la reconversión agroindustrial orientada a las exportaciones. En este sentido, la reconversión y globalización de la industria en Chile, proviene desde atrás, esto es, de su encadenamiento con la globalización del agro chileno, y no de encadenamientos productivos con una industria global.

Esta diferencia no resulta menor a la hora de analizar y entender la dinámica urbana y expansión de ciudades medias. Por de pronto, en el caso de la reconversión industrial más típica, esto es, aquella que se sustenta en la localización de segmentos productivos de una industria globalizada, puede tener un gran potencial de crecimiento y de adopción de nuevas tecnologías, inversión extranjera, y otros supuestos beneficios. Sin embargo, a nivel territorial, configuran más bien enclaves industriales con bajos y a veces nulos encadenamientos territoriales.

Por el contrario, la expansión urbana basada en la agroindustrialización, y en la reconversión productiva del agro en Chile, se manifiesta precisamente, en la configuración de diversos tipos de encadenamientos y articulaciones territoriales entre estas ciudades medias y el mundo agro-rural. En este sentido, el modelo de expansión agroindustrial en Chile, da origen a formas territoriales sustentadas en las diversas formas de las actividades agrarias, que articulan tanto sus formas productivas primarias (agricultura, rural), con sus formas productivas secundarias (agroindustrias, urbanas).

O lo que es lo mismo, mientras en el primer modelo de reconversión industrial, es la ciudad en particular y específica, la que se globaliza, incorporándose como un enclave más dentro de un circuito global de producción industrial, en el caso chileno, basada en al reconversión agroindustrial, es el territorio agrario el que se globaliza, esto es, no sólo su forma urbana (la ciudad agroindustrial), sino también y fundamentalmente, su forma rural (el campo agrícola).

Ahora bien, para ilustrar estas ideas, a continuación presentamos algunos datos empíricos, que nos permiten dimensionar la magnitud y sentido de los cambios en la dinámica urbana y crecimiento de las ciudades medias en Chile en las últimas décadas.

En primer lugar, la dinámica y composición del PIB industrial nos permite visualizar el cambio en el modelo de industrialización, pasando del tradicional modelo sustitutivo de importaciones al de crecimiento agroindustrial. Como puede observarse en la siguiente gráfica, podemos identificar al menos tres etapas en la dinámica de la industrialización chilena en los últimos 50 años. 
- Por un lado, entre 1960 y 1973, que se corresponde con el modelo ISI, y que se refleja en un gran crecimiento del PIB industrial. Entre esos años, el PIB industrial prácticamente se duplicó, pasando de 7.2 mil millones de dólares en 1960, a 14.1 mil millones en 1972. Asimismo, en este periodo, el sector agroindustrial aportaba menos del 25\% de la actividad manufacturera del país.

- Una segunda etapa entre 1973 e inicio de los ochenta. En estos años se da inicio a la reconversión de la manufactura hacia la agroindustria de exportación, provocando el desmantelamiento del aparato industrial que provenía del modelo ISI. En esta fase, prácticamente se frena el crecimiento industrial, habiendo momentos de un gran desplome, como sucedió entre 1973 y 1976, cuando el PIB industrial cayó en casi un 25\% en esos tres años. De hecho, el PIB industrial se mantiene en un nivel de los 12 mil millones de dólares anuales, hasta el año 1985, aproximadamente. En esta fase, el sector agroindustrial, es el menos golpeado, y crece en su participación de un $23 \%$ en 1970, a un $26 \%$ en 1980, y continúa su crecimiento hasta nuestros días.

- Por último, una tercera fase, que corresponde ya al modelo de crecimiento agroindustrial propiamente tal. En esta fase, impulsado por la actividad agroindustrial, el PIB manufacturero retoma su crecimiento, pasando de 12.3 mil millones de dólares en 1985, a más de 33 mil millones en el 2010. En esta fase, veos que el sector agroindustrial pasa de aportar el 26\% del PIB manufacturero total, a casi el 40\% en el 2010, constituyendo así, el sector más dinámica y sobre el cual se sustenta el modelo de industrialización de Chile en las últimas tres décadas.

\section{Ilustración 5. \\ Chile. 1960-2010. PIB manufacturero total y PIB agroindustrial}

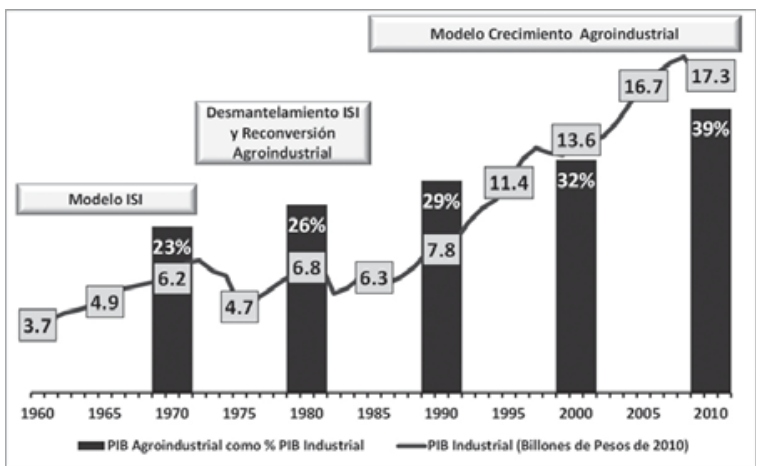

Fuentes: estimaciones propias con base en Banco Central, Indicadores económicos y sociales de Chile, 1960 y 2000, y serie de Cuentas Nacionales, http://si3.bcentral.cl/Siete/secure/cuadros/arboles.aspx 
En términos demográficos, una forma en cómo este proceso de reconversión agroindustrial impacta en la dinámica y expansión de las ciudades medias, es con base en la dinámica del empleo y ocupación, especialmente, en términos del crecimiento del empleo según grandes sectores de actividad, por un lado, y de su distribución urbano-territorial de estas nuevas dinámicas del empleo según sectores económicos.

En otras palabras, el crecimiento económico agroindustrial impulsa el crecimiento del empleo en este tipo de actividades, así como del empleo en actividades conexas (comercio, servicios, transporte, construcción, entre otros), el cual, por las mismas características de la actividad agraria primaria y agroindustrial, ya no se concentra en unas pocas localidades urbanas (ciudades medias, pujantes e integradas a circuitos globales de producción), sino que se distribuye en los territorios agrarios, promoviendo el crecimiento y expansión de todo tipo de poblados urbanos y no pocas localidades rurales, que conforman estos territorios. De esta forma, los factores que pueden explicar las diferencias en el crecimiento y expansión de las ciudades medias en Chile, están en su capacidad para integrarse en territorios agrarios dinámicos, y no tanto en sus condiciones para insertarse en un circuito externo y global de producción manufacturera, o de servicios globales.

En tal sentido, a continuación presentamos información estadística que permite documentar la nueva dinámica y composición del empleo y la ocupación, considerando estos dos grandes ejes: la composición según sectores de actividad, y su distribución según tipos de localidades, diferenciando en este caso, entre ciudades metropolitanas (Santiago, Valparaíso y Concepción), localidades rurales, y las llamadas ciudades medias y pequeñas, que por sus características, optamos por denominar como agrourbes, esto es, localidades urbanas de territorios agrarios ${ }^{6}$.

Esta vinculación entre el nuevo poblamiento agrourbano, y la reconversión agroindustrial en Chile, se da puede analizar a través del cambio en la composición de la ocupación según grandes sectores de actividad económica. Al respecto, podemos identificar cuatro dinámicas diferentes.

- Por un lado, sectores con un alto crecimiento del empleo. Corresponde al sector de servicios financieros y otros a empresas, y a la construcción, los cuales crecieron en un 450\% y 330\%, entre 1982 y el 2009, respectivamente. En el primer caso, se trata de servicios muy dinámicos, pero con poca capacidad de crecimiento autosustentable, pues corresponden en general a actividades de soporte y apoyo a actividades productivas. Algo similar puede decirse de la construcción, la cual es una actividad económica muy dependiente de la dinámica del resto de la economía.

- Por otro lado, identificamos a la actividad agroindustrial, el comercio y transporte y comunicaciones, los cuales crecen muy por encima del promedio nacional, prácticamente duplicándose entres esos mismo años. De ellos, sólo la agroindustria corresponde a un 
sector productivo propiamente tal, con capacidad de crecimiento autosustentable. Por el contrario, el comercio y el transporte, son actividades cuya dinámica depende directamente del auge o estancamiento de otros sectores económicos, y particularmente, de los sectores productivos propiamente tales.

- En tercer lugar, identificamos los sectores de provisión de servicios de electricidad, gas y agua, servicios sociales y la minería, los cuales crecen prácticamente muy similar al promedio nacional. Aquí resulta interesante la dinámica de la minería, pues es la principal fuente de divisas del país, y de sustento de la dinámica y crecimiento económico nacional.

- Finalmente, ubicamos los servicios personales, la manufactura no agroindustrial y la agricultura, los cuales crecen muy por debajo del promedio nacional.

De estos datos, lo relevante es que entre los sectores productivos propiamente tales, es la agroindustria la que presenta el mayor ritmo de crecimiento en la ocupación de la fuerza de trabajo en Chile. De esta forma, vemos que el modelo chileno se sustenta en dos grandes actividades productivas, ambas orientadas a la exportación, y derivadas o articuladas actividades primario-extractivas. Por un lado, la tradicional actividad minera, en especial de extracción cuprífera. Por otro lado, vemos la emergencia de la actividad agroindustrial, la que si bien implica un nivel de procesamiento y agregación de valor, se trata sin duda de una actividad directamente vinculada a la agricultura. En otras palabras, hoy más que nunca, en su base económica, Chile sigue siendo un país agrícola-minero.

Ilustración 6.

Chile, 1982-2009. Crecimiento de la ocupación, según grandes sectores de actividad económica.

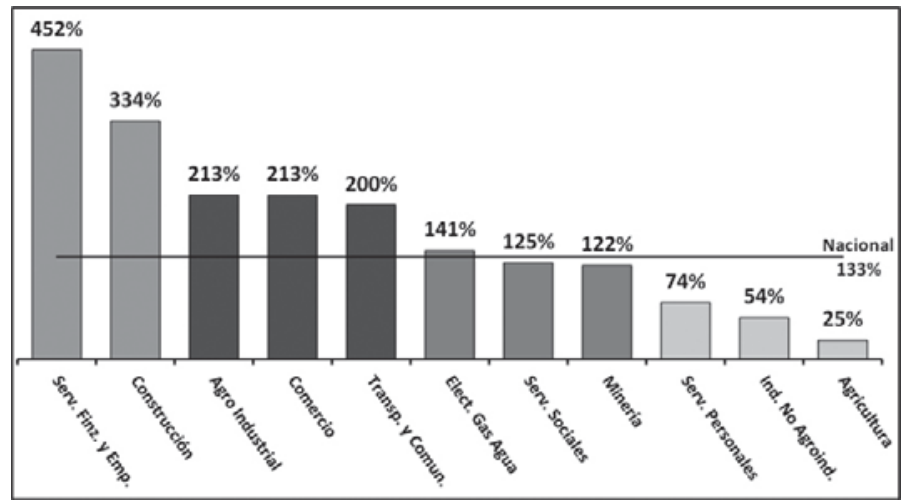

Fuentes: estimaciones propias con base en Censo de Población, 1982, y Encuesta CASEN, 2009. 
Ahora bien, considerando los sectores más dinámicos en la generación de empleos, vemos una diferencia sustantiva en términos de la localización y asentamiento de la fuerza de trabajo que emplean cada uno de ellos. En efecto, mientras las actividades de servicios financieros y a empresas, tienden a localizarse preferentemente en las ciudades metropolitanas, y de manera particular en la ciudad de Santiago, los trabajadores de la agroindustria se asientan en cambio, en una amplia gama de agrourbes y asentamientos rurales que pueblan los diversos territorios agrarios del país.

En efecto, como se ilustra en la gráfica anterior, vemos que prácticamente el 70\% del crecimiento del empleo en servicios financieros y a empresas, se localiza actualmente en las ciudades metropolitanas, mientras que sólo el 26\% del crecimiento se asienta en las agrourbes.

Por el contrario, en el caso del empleo agroindustrial, vemos que sólo un tercio de los nuevos puestos de trabajo en este tipo de actividad, se localizan en las ciudades metropolitanas, mientras que los dos tercios restantes se ubican en los territorios agrarios, ya sea en sus formas de poblados rurales (21\%), o bien en las agrourbes (46\%).

Ilustración 7.

Chile, 1982-2009. Crecimiento de la ocupación, según sector económico, y localidad de residencia de la fuerza de trabajo.
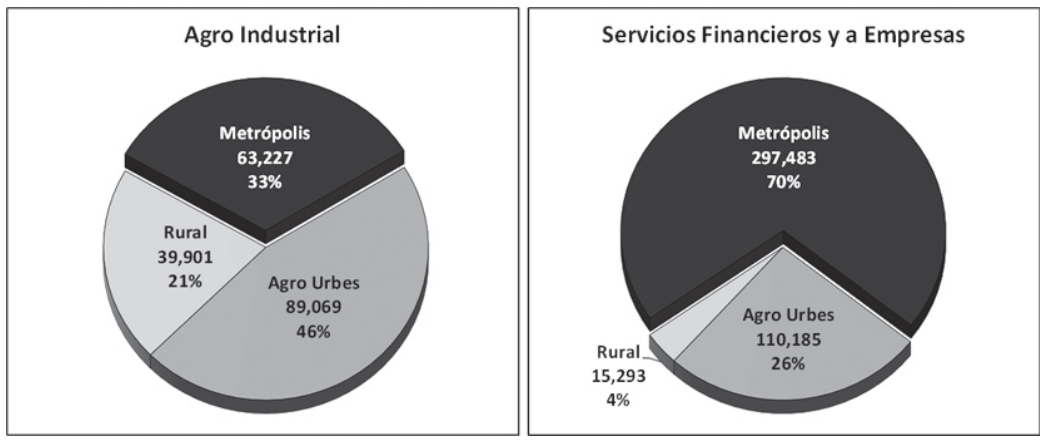

Fuentes: estimaciones propias con base en Censo de Población, 1982, y Encuesta

CASEN,2009.

No se trata de una diferencia menor. Indica por un lado, el tipo de reconversión económica y ocupacional que tiene actualmente las ciudades metropolitanas, particularmente Santiago. Se trata de una ciudad que se reconvertido desde las actividades manufactureras de antaño, hacia un sector moderno y dinámico, pero que sustenta su crecimiento en la actividad productiva tanto de la minería del cobre, como de la agricultura moderna y la agroindustria de exportación. 
Por otro lado, los datos reafirman nuestra tesis de que en el caso de Chile, la expansión urbana de ciudades medias, se corresponde más con su reconversión agroindustrial, y la conformación de territorios agrarios dinámicos. En particular, a diferencia de otros contextos de expansión urbana de ciudades medias en América Latina, en el caso de Chile resulta más bien una expansión urbana con base en el crecimiento, modernización y reconversión productiva de los territorios agrarios.

\section{Ciudades medias y agrourbes en Chile}

Una forma de ilustrar esta nueva dinámica y caracterización de las ciudades medias como agrourbes, es con base en su especialización productiva. Al respecto, presentamos una comparación entre las agrourbes, y las ciudades metropolitanas, como una forma de ilustrar sus diferencias. Posteriormente, hacemos un análisis que permite cómo el crecimiento demográfico está directamente relacionado con la reconversión agroindustrial de las ciudades medias en Chile.

Respecto al primer punto, vemos que a diferencia de las ciudades metropolitanas, las agrourbes sí muestran una especialización agroindustrial (un valor del índice superior a 1, indica especialización en ese sector, y un valor menor que 1 , indica lo contrario). Asimismo, vemos que en el caso de las agrourbes, la razón entre el empleo agroindustrial, y el empleo en el resto de la industria manufacturera, es muy superior al valor de este índice que prevalece en las ciudades metropolitanas.

Estos datos nos indican que efectivamente, las agrourbes muestran una especialización de tipo agroindustrial, y no de otro tipo de actividad manufacturera, a diferencia de las ciudades metropolitanas, donde además de no darse una especialización agroindustrial, son otros sectores manufactureros los de mayor peso relativo en la generación de empleo.

\section{Ilustración 8. \\ Chile, 2009. Índice de especialización agroindustrial global, e Índice de especialización agroindustrial en la manufactura}

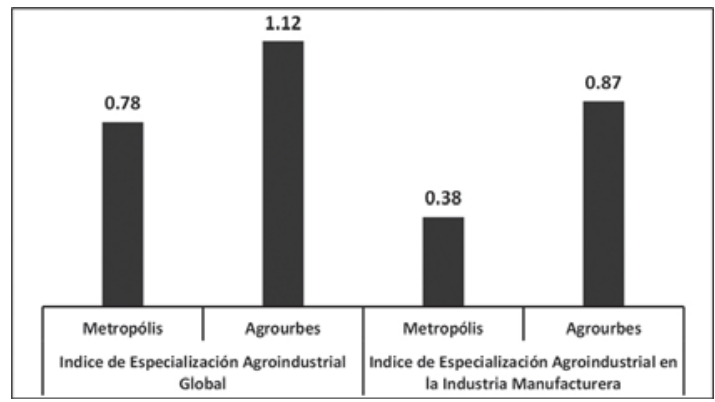

Fuentes: estimaciones propias con base en Encuesta CASEN,2009. 
En relación al segundo punto, vemos que en las regiones donde la reconversión agroindustrial y modernización agraria es mayor y más dinámica, es donde se da un mayor crecimiento demográfico y expansión de las agrourbes. En efecto, como se ilustra en la siguiente gráfica, vemos que es la región Central de Chile (regiones de Coquimbo a Talca), donde se da preferentemente la expansión urbana de las agrourbes. Por un lado, en esta región las agrourbes muestran un mayor dinamismo demográfico, con una tasa de crecimiento del 2.7\% anual promedio entre 1982 y el 2009, muy superior no sólo al promedio nacional, sino al de las demás ciudades medias, tanto del norte como del sur y región austral del país. Por otro lado, es la zona de especialización agroindustrial por excelencia, con índices muy superiores al promedio nacional.

Por el contrario, en las regiones de las zonas norte y sur y austral del país, la reconversión agroindustrial es significativamente menor, a la vez que también se da un menor impulso demográfico de las ciudades medias y agrourbes. Esto indica que el crecimiento demográfico de las ciudades medias en realidad se circunscribe territorialmente, a la región de mayor dinamismo agroindustrial, y que corresponde a la región con mayor vocación agraria del país.

\section{Ilustración 9. \\ Chile, Crecimiento demográfico de agrourbes según zona geográfica (1982-2009), e índice de Especialización Agroindustrial de las agrourbes, según zona geográfica}
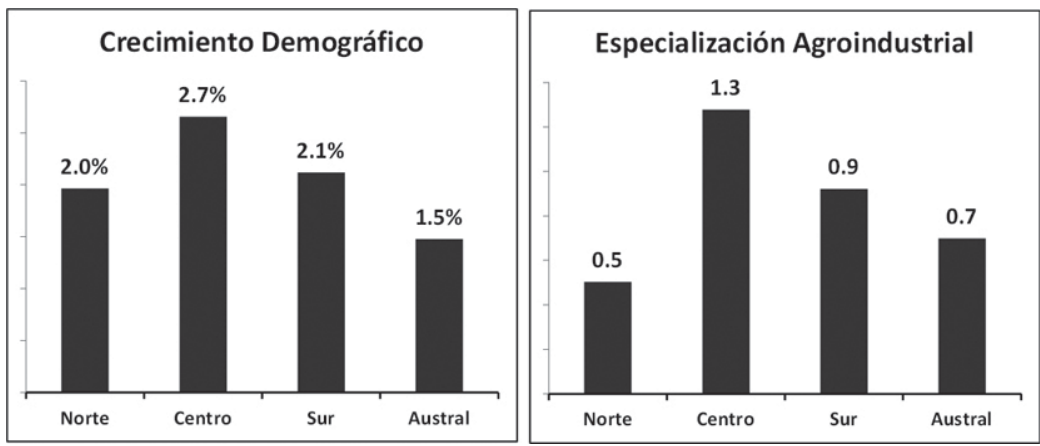

Fuentes: estimaciones propias con base en Censo de Población, 1982, y Encuesta CASEN,2009.

En la región norte, en cambio, el auge demográfico es menor, y está sustentado en la actividad minera, así como por el impulso estatal a determinadas ciudades con base en principios y lógicas geopolíticas, que sustentan su poblamiento. 
El Sur en realidad corresponde a una zona heterogénea. Por un lado, esta la región de Valdivia y Osorno, con un gran impulso agroindustrial y piscisilvoagropecuario. En esta región se da un mayor crecimiento demográfico y mayor impulso agroindustrial. Por el otro lado, en esta zona sur del país, se ubica también la región de Temuco y la Araucanía, que corresponden a la zona de asentamiento mapuche, misma que se encuentra en gran medida, alejada de los procesos de reconversión agropecuaria y agroindustrial.

\section{Conclusiones}

En este texto hemos querido discutir la tesis de que en Chile se habría configurado un modelo de expansión urbana y de ciudades medias diferentes al esperado y que se observa en ciertos países de la región. En particular, hemos documentado que en el caso de Chile, el crecimiento de las ciudades medias no esta vinculado ni un proceso de descentralización y desconcentración metropolitana, ni a un proceso de relocalización industrial de segmentos productivos, en el marco de una inserción en procesos de producción industrial globales.

Por el contrario, el crecimiento de las ciudades y localidades urbanas de rango intermedio se debería al impulso de un modelo de urbanización completamente diferente, sustentado en la modernización productiva y globalización económica del agro chileno. Se trata del desarrollo de agrourbes, de un proceso de urbanización agraria, en el marco de la configuración de espacios agropolitanos.

En tal sentido, no es posible hablar, en el caso chileno, de ciudades medias en el sentido clásico de ciudades industriales, o similares, sino más bien, habría que referirse a estas como ciudadesagrarias, o bien como agrourbes, esto es, como el componente urbano de los territorios agrarios en el Chile contemporáneo. Por lo mismo, no son ciudades que compitan directamente con las metrópolis en la localización industrial y de actividades de servicios y de apoyo, sino, que forman parte de una nueva configuración urbana, que se yuxtapone a la configuración urbano metropolitana tradicional.

Se trata de ciudades que su inserción en la globalización, es desde atrás, esto es, de su integración a la reconversión y modernización del agro chileno, y no desde adelante, esto es, como parte de un proceso global de producción industrial. Se trata no sólo de una diferencia semántica, sino que tiene importantes implicaciones demográficas, económicas y territoriales. Por de pronto, se trata de ciudades que por su dinámica económica, se encuentran fundamentalmente afincadas con los territorios agrarios, conformando un mismo espacio económico y social.

$\mathrm{Al}$ respecto, los datos presentados nos permiten mostrar que efectivamente, en Chile su inserción en la economía global se da con base en la 
reconversión productiva del agro y el impulso de la actividad agroindustrial, y no por un proceso de reconversión de otros segmentos de la industria manufacturera.

Asimismo, hemos mostrado que esta reconversión agroindustrial ha modificado también el entorno laboral de las ciudades intermedias, promoviendo e impulsando el empleo en este tipo de actividades.

Por último, también hemos mostrado que son precisamente las agrourbes más vinculadas a la reconversión agroindustrial, la que han experimentado el mayor dinamismo demográfico, como consecuencia entre otros aspectos, de la concentración en ellas del empleo agroindustrial, así como de otras actividades subsidiarias (comercio, transporte, comunicaciones, construcción). 


\section{Notas}

${ }^{1}$ Estimaciones con base en los censos de población de 1907 y 2002.

${ }^{2}$ El otro pilar del modelo chileno, es la modernización de la actividad minera de exportación, sustentada básicamente en la explotación del cobre.

${ }^{3}$ Sin duda, en Chile también podemos identificar ciudades medias que no forman parte de espacios agrarios, pero además de ser relativamente pocas, corresponden en general a ciudades mineras, ciudades geopolíticas del norte y extremo sur del país, y las ciudades turísticas del litoral central.

${ }^{4}$ Los datos para el 2010 se refieren a las proyecciones de población que realiza el INE, pues el censo más reciente se levantó en el 2002, y actualmente entre abril y junio de este año (2012), se está levantando el XVIII censo de población.

${ }^{5}$ La RMS, está formada por las comunas no costeras que conformaban la provincia de Santiago, y que incluyen no sólo la ciudad de Santiago, principal metrópolis chilena, sino también, un importante hinterland rural y agrario.

${ }^{6}$ Cabe señalar, que entendemos que no todas las ciudades medias conforman necesariamente agrourbes, así como también que no todas las agrourbes son igualmente dinámicas y en expansión demográfica y económica. Sin embargo, en esta ocasión, nos centraremos en las tendencias globales, dejando para otros textos un análisis más detallado de estas diferencias. 


\section{Bibliografía}

Alegría, Tito, Jorge Carrillo y Jorge Alonso (1997), “Reestructuración productiva y cambio territorial: un segundo eje de industrialización en el norte de México”. Revista de la CEPAL, n. 61, pp. 187-204. Comisión Económica para América Latina y el Caribe, Santiago, Chile.

Aguilar, A.; B. Graizbord y A. Sánchez (1996), Las ciudades intermedias y el desarrollo regional en México. CONACULTA-UNAM-El Colegio de México, México.

Atienza, Miguel y Aroca, Patricio (2012), “Concentración y crecimiento en Chile: una relación negativa ignorada”. EURE. vol.38, n.114, pp. 257277.

Berdegué, J.; B. Jara; R. Fuentealba; J. Tohá; F. Modrego; A. Shejtman y N. Bro. (2011a), Territorios funcionales en Chile. Documento de Trabajo No. 102. Programa Dinámicas Territoriales Rurales. RIMISP, Santiago, Chile.

Berdegué, J.; Jara, E.; Modrego, F.; Sanclemente, X.; Sheitman, A. (2011b), “Ciudades Rurales en Chile”. Revista Paraguaya de Sociología N 138. Centro Paraguayo de Estudios Sociológicos.

Canales, Alejandro I. (1996), “Cambio agrario y poblamiento regional en Chile”. Estudios Demográficos y Urbanos 31. Vol. 11, No.1. Enero - Abril. El Colegio de México. Págs. 173 - 196, México.

Canales, Manuel (2008), “Agrópolis-metrópolis. Más allá de lo rural y lo urbano”. Congreso de Desarrollo Rural, IICA, 2008 Santiago, Chile. (En www.IICA.cl)

Canales Cerón, Manuel, y Alejandro I. Canales (2012), “La Nueva Provincia: (re)poblamiento de los territorios agrarios. Chile 1982-2002”. Revista Anales, Séptima Serie, No 3, julio 2012 (fundada en 1844), Universidad de Chile. Págs. 155-173, Santiago, Chile.

Canales, Manuel y María Cristina Hernández (2011), “Nueva agricultura y geografía humana. Refundación y dinamismo de las agro-urbes”. Revista Paraguaya de Sociología N 138. Centro Paraguayo de Estudios Sociológicos.

CEPAL, 2012. Población, territorio y desarrollo sostenible. Naciones Unidas, Chile. LC/L.3475(CEP.2/4).

Cunha, J.M. (2002), Urbanización, redistribución espacial de la población y transformaciones socioeconómicas en América Latina, Serie Población y desarrollo, No 30 (LC/L.1782-P), CEPAL, Santiago de Chile. Publicación de las Naciones Unidas, $\mathrm{N}^{\circ}$ de venta: S.02.II.G 97. 
Cunha, J.M. y J. Rodríguez (2009), “Crecimiento urbano y movilidad en América Latina”. Revista Latinoamericana de Población, núm. 4-5, enero-diciembre, pp. 27-64

de Mattos, Carlos A. (2002), “Transformación de las ciudades latinoamericanas: ¿Impactos de la globalización?”. EURE (Santiago) [online]. 2002, vol.28, n.85 [citado 2012-12-17], pp. 5-10. Disponible en: <http:// www.scielo.cl/scielo.php? script=sci_arttext \&pid=S025071612002008500001\&lng=es\&nrm=iso>.

de Mattos, Carlos A. (1998), “Reestructuración, globalización, nuevo poder económico y territorio en el Chile de los noventa”. En Carlos A. de Mattos, Daniel Hiernaux Nicolás y Carlos Restrepo Botero (Comps.) Globalización y Territorio. Impactos y perspectivas. Pontificia Universidad Católica de Chile, Instituto de Estudios Urbanos, y Fondo de Cultura Económica.

Diniz, Clélio Campolina y Marco Aurélio Crocco (1998), “Reestructuración económica e impacto territorial: el nuevo mapa de la industria brasileña”. En Carlos A. de Mattos, Daniel Hiernaux Nicolás y Carlos Restrepo Botero (Comps.) Globalización y Territorio. Impactos y perspectivas. Pontificia Universidad Católica de Chile, Instituto de Estudios Urbanos, y Fondo de Cultura Económica.

Garza, Gustavo (2003), La urbanización de México en el siglo XX. CEDDU, El Colegio de México, México.

Geisse, Guillermo (1983), Economía y política de la urbanización en Chile. El Colegio de México y PISPAL, México.

Gwinne Robert y Cristóbal Kay (1997), “Agrarian Change and the Democratic Transition in Chile: an Introduction”. Bulletin of Latin American Research. Volume 16, Issue 1, pages 3-10, January.

Hiernaux, D. (1998), "Restructuración económica y cambios territoriales en México”. En Carlos A. de Mattos, Daniel Hiernaux Nicolás y Carlos Restrepo Botero (Comps.) Globalización y Territorio. Impactos y perspectivas. Pontificia Universidad Católica de Chile, Instituto de Estudios Urbanos, y Fondo de Cultura Económica.

Kay, Cristóbal (2002), “Agrarian reform and the neoliberal counter-reform in Latin America”. En Jacquelyn Chase (Ed.), The Spaces of Neoliberalism: Land, Place and Family in Latin America. Kumarian Press Inc. USA.

Lufin Varas, Marcelo y Atienza Ubeda, Miguel (2010), “Diferencias entre la composición sectorial y ocupacional de las principales ciudades chilenas”. EURE. vol.36, n.108, pp. 75-93, Chile.

PNUD (2008), Desarrollo Humano en Chile Rural. Seis millones por nue- 
vos caminos. Programa de las Naciones Unidas para el Desarrollo. Santiago, Chile.

Pradilla Cobos, Emilio (1993), Territorios en crisis. México 1970-1992. Red Nacional de Investigación Urbana y Universidad Autónoma Metropolitana, Unidad Xochimilco, México.

Rodríguez V., Jorge y Gustavo Busso (2009), Migración interna y desarrollo en América Latina entre 1980 y 2005. Un estudio comparativo con perspectiva regional basado en siete países. CEPAL; Naciones Unidas, Chile.

Rodríguez, J.; González, D.; Ojeda, M.; Jiménez, M.; Stang, F. (2009), “El sistema de ciudades chileno en la segunda mitad del siglo XX: entre la suburbanización y la desconcentración”. Estudios Demográficos y Urbanos, Enero-Abril, 7-48. Número 2, Vol (24), año 2009

Sobrino, Luis Jaime (2002), “Globalización, crecimiento manufacturero y cambio en la localización industrial en México”. Estudios Demográficos y Urbanos, Núm. 49, pp. 5-38.

Soto, Alba Celina, Luis Huesca Reynoso y María del Carmen Hernández Moreno (2008), "El modelo de crecimiento de la frontera norte de México a partir del TLCAN”. Análisis Económico, Núm. 54, vol. XXIII. México.

Villa, Miguel (1992), “Urbanización y transición demográfica en América Latina: una reseña del período 1930-1990”, en IUSSP, El Poblamiento de las Américas, Vol. 2, pp.339-356. IUSSP-SOMEDE-ABEP-FCD-PAAPROLAP, México. 